\title{
Investigating heat transfer augmentation using gradient heat flux measurement and PIV method
}

\author{
Andrey Mityakov ${ }^{*}$, Aleksandr Babich, Aleksandr Bashkatov, Andrey Gusakov, \\ Aleksey Dymkin, Elza Zainullina, Sergey Sapozhnikov, Vladimir Mityakov and \\ Vladimir Seroshtanov \\ Deparment of Thermophysics of Power Units, Peter the Great St. Petersburg Polytechnic University, \\ Russian Federation, 195251, Saint-Petersburg
}

\begin{abstract}
The heat transfer and the fluid flow behaviour at the surface of a circular cylinder with and without turbulizing rods, heated by saturated steam rectangular and circular fins were studied for different Reynolds numbers. The proposed approach of simultaneous use of gradient heat flux measurement and PIV method allowed us optimizing the geometry of the system with the rods and increasing heat transfer coefficient up to $20 \%$.
\end{abstract}

\section{Introduction}

Up to the present time, it was not possible to combine real-time fluid field with local heat flux in problems of heat flux augmentation. The combining of gradient heat flux measurement [1] and PIV method of fluid visualization [2] gives new opportunities for investigation [3], [4]. Gradient heat flux sensors (GHFS) allow measuring heat flux per unit area practically without inertia because of their response time constant of $10^{-8}$ to $10^{-9} \mathrm{~s}$. Therefore, we can research both processes simultaneously.

\section{Experimental details and results}

Experiments were carried out in the closed-circuit wind tunnel. The tube has test section of $870 \mathrm{~mm}$ length and contraction cone outlet diameter of $450 \mathrm{~mm}$. The Eiffel chamber is made of plexiglas to allow PIV measurements from outside the chamber. The wind tunnel is equipped with a water-air heat exchanger to maintain constant flow temperature. Freestream temperature $T_{\infty}$ and velocity $W_{\infty}$ were measured by the Testo $435-2$ multifunction HVAC and IAQ Meter. The longitudinal turbulent intensity was found to be less than $0.5 \%$ for the $10^{4}<\operatorname{Re}<10^{5}$. In the current study we used the method of simultaneous PIV and heat flux measurement.

To obtain a 3D velocity field stereo PIV of POLIS type was used [2]. The flow was seeded by the smoke with a particle size of about $3 \mu \mathrm{m}$ in diameter. ActualFlow software application was used for data processing.

*Corresponding author: andrey.mityakov@gmail.com 
The heat flux was measured by gradient heat flux sensors (GHFS) produced of a single crystal of $99.99 \%$ pure bismuth in Peter the Great St. Petersburg Polytechnic University (Russia). The GHFS plan form dimensions were $2 \times 2 \mathrm{~mm}$, while its thickness was about $0.2 \mathrm{~mm}$.

The cylinder model, used in the experiments was made of a steel sheet of $0.1 \mathrm{~mm}$ in thickness, its diameter was of $66 \mathrm{~mm}$, and its length was of $800 \mathrm{~mm}$. The model was heated by saturated water steam with temperature close to $100^{\circ} \mathrm{C}$. Experiments were carried out with constant temperature boundary conditions which were confirmed by the infrared measuring using FLIR P640 infrared camera. The GHFS was installed at the cylinder surface flat with it. The cylinder was turned about its axis by an electric driver which allowed moving the sensor in circumferential direction along the cylinder surface.

Figure 1 illustrates the scheme of our experiment. Attack angle $\beta$ is defined as an angle between the laser sheet and a cylinder axis $\left(\beta=90^{\circ}\right.$ for the cross-flow). The GHFS angular coordinate $\varphi$ is defined as the angular distance between the front stagnation point and GHFS location. Flow velocity component $W_{z}$ characterizes the flow three-dimensionality. More detailed information about the method could be found in the work [5].

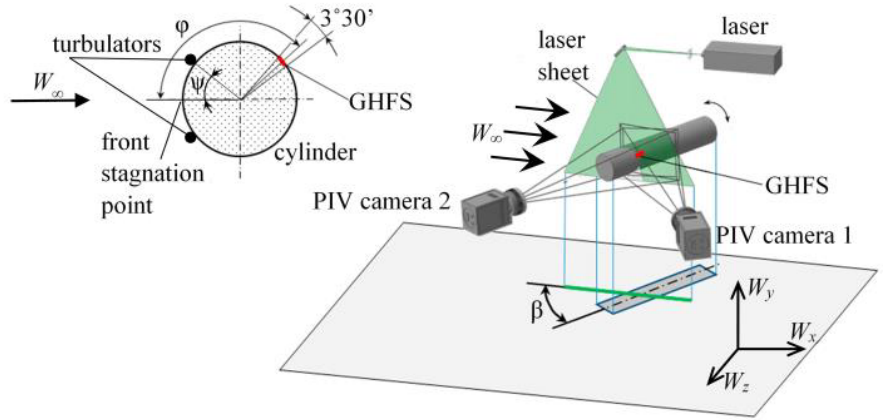

Fig. 1. Scheme of the experiment with cylindrical model

In the first series of experiments the circular cylinder with turbulizing rods was obtained. The rods with diameter $d_{T}$ were placed parallel to the axis of the cylinder, with a gap (Figure 2, b) $\delta$ and without it (Figure 2, a). The rods were placed symmetrically from the front stagnation point at the angle $\psi$. The values of $d_{T}, \delta$ and $\psi$ were optimized during the experiment.

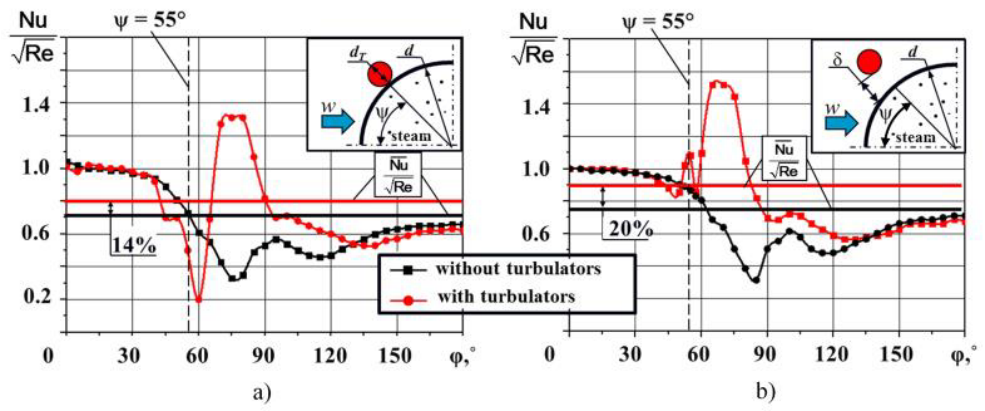

Fig. 2. Heat transfer enhancement with turbulators $\left(d_{T}=1 \mathrm{~mm}\right.$ for $\mathrm{Re}=4.9 \cdot 10^{4}, \beta=90^{\circ}, \psi_{\mathrm{T}}=55^{\circ}$ : $\mathrm{a}$ - without the gap; b - with the gap, $\delta=1.2 \mathrm{~mm}$.

Figure 2 shows curves of heat transfer coefficient when $\beta=90^{\circ}$. The local heat transfer coefficient near the rods increases 2.5 times, and the average heat transfer coefficient increases by 14 to $20 \%$ in comparison of the cylinder without turbulence rods (black line). 
PIV technology visualized the velocity field near the model surface (Figure 3) and allowed finding optimal geometry of the system.

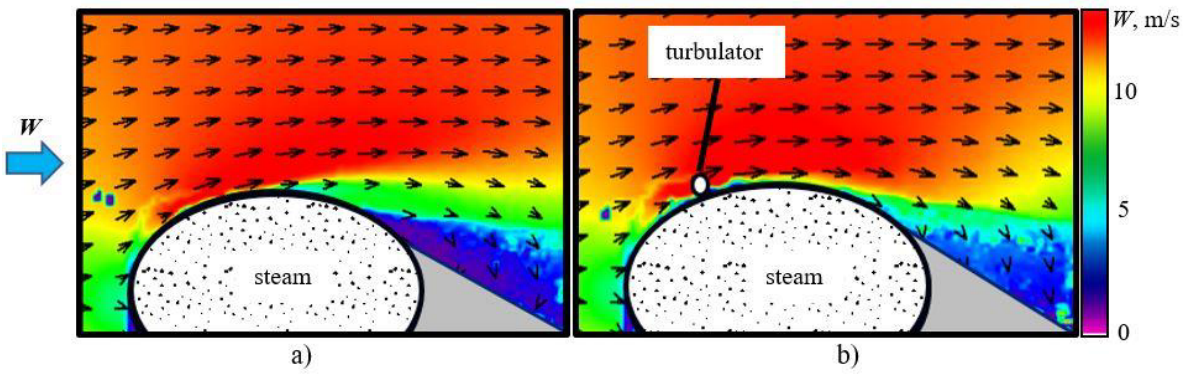

Fig. 3. Visualisation of flow around the cylinder with the turbulizing rods (b) and without them (a). $\operatorname{Re}=5.3 \cdot 10^{4}, \beta=60^{\circ}, \psi_{\mathrm{T}}=63^{\circ}, \mathrm{d}_{\mathrm{T}}=1 \mathrm{~mm}$

Another series of experiments was devoted to investigation of the flow around a circular cylinder at $\beta<90^{\circ}$. The turbulizing rods were placed close to the cylinder surface or with some gap from it. It was shown that at different values of $\beta$ the optimal value of $\psi$ was changed.

The next stage of research was devoted to the flow around isothermal ("ideal") rectangular (Figure 4) and circular (Figure 5) fins which were mounted normally to the circular cylinder surface.

Two GHFSs with sizes $4 \times 7 \mathrm{~mm}$ and $2 \times 2 \mathrm{~mm}$ and with the thickness of $0.2 \mathrm{~mm}$ were installed at the fins. The volt-watt sensitivity of these sensors was $12.3 \mathrm{mV} / \mathrm{W}$ and 21.8 $\mathrm{mV} / \mathrm{W}$, respectively. The sensors were installed in different places along the length of the fin. The fin was set in three positions: upstream (Figure 4, a), normal to the flow (Figure 4, b) and downstream (Figure 4, c).
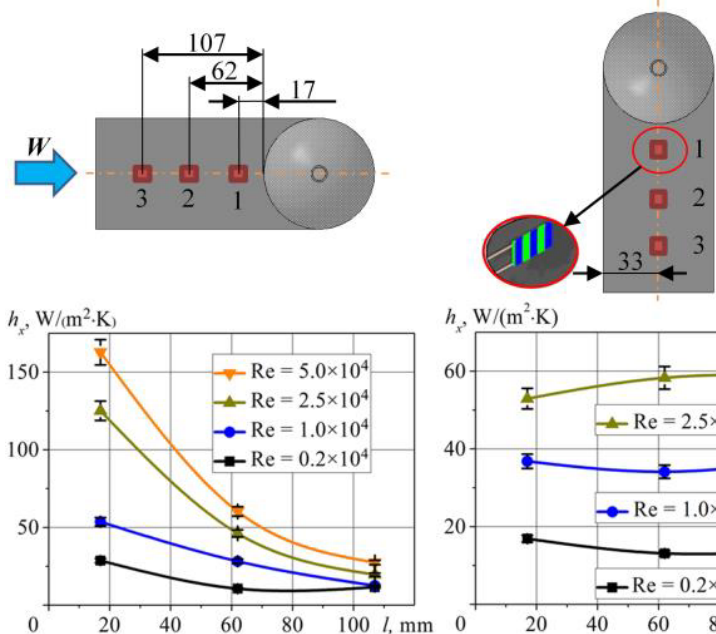

a)

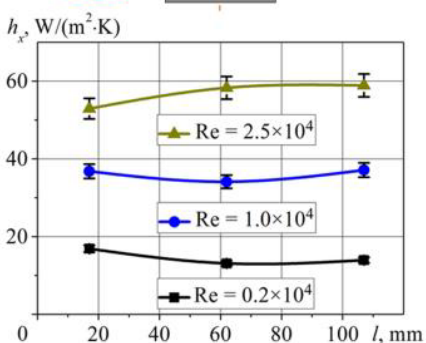

b)
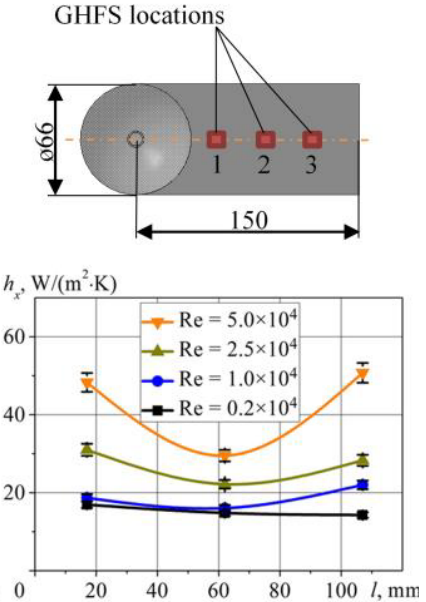

c)

Fig. 4. Rectangular fin orientation within the flow and the local heat transfer coefficients on its surface: $\mathrm{a}$ - upstream, $\mathrm{b}$ - normal to the stream, $\mathrm{c}$ - downstream

The velocity field and heat flux were fixed for some real time moments. Relationship between the local heat transfer coefficient and Reynolds number for fin installed in different positions are shown in Figure 4. The Standard Relative Uncertainty was evaluated in accordance with type B. 
Non-monotonic character of the curves in Figure 4 attracts deserves attention and requires further investigation. Next step was to associate heat flux measurement with velocity field.

One of our GHFSs was installed at the surface of the circular fin (Figure 5, a). During the experiment, the model was rotated around the axis of the cylinder. The dependence of the local heat transfer coefficient, azimuthal angle and Reynolds number is presented in Figure 5, b.

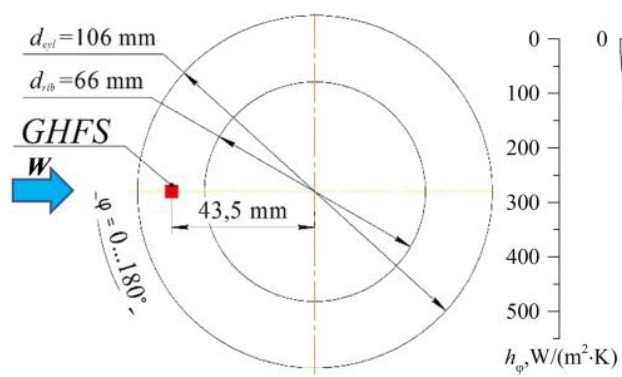

a)

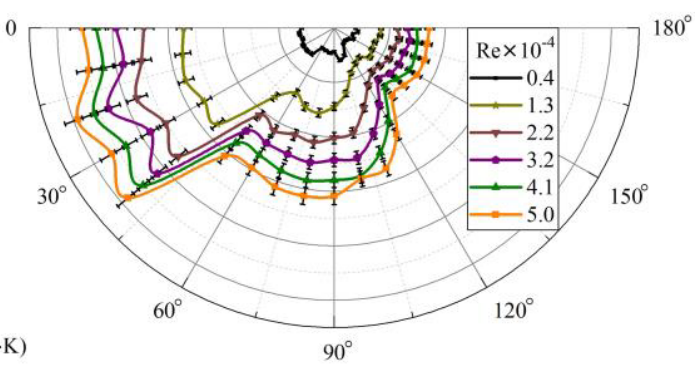

b)

Fig. 5. Location of GHFS at the circular fin (a) and relations between local heat transfer coefficient, azimuthal angle and Reynolds number (b)

\section{Conclusion}

The combination of PIV method and the gradient heat flux measurement allowed investigating the flow and heat transfer at the surface of circular cylinder with turbulizing rods and with rectangular and circular fins. The geometry of the system with the rods was optimized during the experiment. Moreover, it was the first step in the comparison of heat flux and fluid flow in the same moments of time during the process.

\section{References}

1. V. Mityakov, S.Z. Sapozhnikov, V.Y. Mityakov et al., Sensors and Actuators A: Physical 176, 1 (2012)

2. «POLIS» velocity profile measuring instrument ([website]/Institute of Thermophysics SB RAS, Novosibirsk - access mode: http://eng.polis-instruments.ru/)

3. V.V. Seroshtanov, A.V. Bashkatov, E.R. Zaynullina et al., Industrial and Medical Measurement and Sensor Technology Vehicle Sensor Technology 5, 26 (2016)

4. A.V. Bashkatov, A.Y. Babich, A.A. Gusakov et al., Industrial and Medical Measurement and Sensor Technology Vehicle Sensor Technology 5, 15 (2016)

5. S.Z. Sapozhnikov, V.Y. Mityakov, A.V. Mityakov et al., Appl. Mech. Materials 629, 444 (2014) 